BONN-TH-97-10

SFB-375/295, TUM-HEP-311/98

TU-531, RCNS-97-05

January 1998

\title{
Supersymmetry Breakdown at a Hidden Wall
}

\author{
H. P. Nilles ${ }^{1,2}$, M. Olechowski ${ }^{3, *}$ AND M. Yamaguchi ${ }^{4}$ \\ ${ }^{1}$ Physikalisches Institut, Universität Bonn \\ Nussallee 12, D-53115 Bonn, Germany \\ ${ }^{2}$ Max-Planck-Institut für Physik \\ D-80805 München, Germany \\ ${ }^{3}$ Physik Department, Technische Universität München \\ D-85747 Garching, Germany \\ ${ }^{4}$ Department of Physics, Tohoku University \\ Sendai 980-77, Japan
}

\begin{abstract}
We consider hidden sector supersymmetry breakdown in the strongly coupled heterotic $E_{8} \times E_{8}$ theory of Hořava and Witten. Using effective field theory methods in four dimensions, we can show that gravitational interactions induce soft breaking terms in the observable sector that are of order of the gravitiono mass. We apply these methods to the mechanism of gaugino condensation at the hidden wall. Although the situation is very similar to the weakly coupled case, there is a decisive difference concerning the observable sector gaugino mass; with desirable phenomenological as well as cosmological consequences.
\end{abstract}

* On leave of absence from Institute of Theoretical Physics, Warsaw University, Poland. 


\section{Introduction}

From all the new and interesting results in string dualities, it is the heterotic M-theory of Hořava and Witten [1] that seems to have immediate impact on the discussion of the phenomenological aspects of these theories. One of the results concerns the question of the unification of all fundamental coupling constants [2] and the second one the properties of the soft terms (especially the gaugino masses) once supersymmetry is broken [3]. In both cases results that appeared problematic in the weakly coupled case get modified in a satisfactory way, while the overall qualitative picture remains essentially unchanged.

The heterotic M-theory is an 11-dimensional theory with the $E_{8} \times E_{8}$ gauge fields living on two 10-dimensional boundaries (walls), respectively, while the gravitational fields can propagate in the bulk as well. A $d=4$ dimensional theory with $N=$ 1 supersymmetry emerges at low energies when 6 dimensions are compactified on a Calabi-Yau manifold. The scales of that theory are $M_{11}$, the $d=11$ Planck scale, $R_{11}$ the size of the $x^{11}$ interval, and $V \sim R^{6}$ the volume of the Calabi-Yau manifold. The quantities of interest in $d=4$, the Planck mass, the GUT-scale and the unified gauge coupling constant $\alpha_{G U T}$ should be determined through these higher dimensional quantities. The fit of ref. [2] identifies $M_{G U T} \sim 3 \cdot 10^{16} \mathrm{GeV}$ with the inverse Calabi-Yau radius $R^{-1}$. Adjusting $\alpha_{G U T}=1 / 25$ gives $M_{11}$ to be a few times larger than $M_{G U T}$. On the other hand, the fit of the actual value of the Planck scale can be achieved by the choice of $R_{11}$ and, interestingly enough, $R_{11}$ turns out to be an order of magnitude larger than the fundamental length scale $M_{11}^{-1}$. A satisfactory fit of the $d=4$ scales is thus possible, in contrast to the case of the weakly coupled heterotic string, where the string scale seemed to be a factor 20 larger than $M_{G U T}$.

Otherwise the heterotic $E_{8} \times E_{8}$ string looks rather attractive from the point of view of phenomenological applications. One seems to be able to accommodate the correct gauge group and particle spectrum. The mechanism of hidden sector gaugino condensation leads to a breakdown of supersymmetry with vanishing cosmological constant to leading order. With a condensate scale $\Lambda \sim 10^{13} \mathrm{GeV}$, one obtains a gravitino mass in the $\mathrm{TeV}$ range and soft scalar masses in that range as well. In the simplest models [4, 5, 6] this type of supersymmetry breakdown is characterized through the vacuum expectation value of moduli fields other than the dilaton, giving a small problem with the soft gaugino masses in the observable sector: they turn out to be too small, generically some two orders of magnitude smaller than the soft scalar masses. It is again in the framework of heterotic M-theory that this problem is solved [3]; gaugino masses are of the same size as (or even larger than) the soft scalar masses.

The mechanism of hidden sector gaugino condensation itself can be realized in a way very similar to the weakly coupled case. This includes the mechanism of cancellation of the vacuum energy, which in the weakly coupled case arises because of a cancellation of the gaugino condensate with a vacuum expectation value of the three index tensor field $H$ of $d=10$ supergravity. This cancellation is at the origin of the fact that supersymmetry breakdown is dominated by a $T$ modulus field rather than the dilaton $(S)$. Hořava [7] observed that this compensation of the vacuum expectation values of the condensate and $H$ carries over to the M-theory case. In [3] we explicitly worked out the mechanism of gaugino condensation in the heterotic M-theory and showed the 
similarity to the weakly coupled case. Now the gaugino condensate forms at the hidden 4-dimensional wall and is cancelled locally at that wall by the vacuum expectation value (vev) of a Chern-Simons term. This also clarifies some questions concerning the nature of the vev of $H$ that arose in the weakly coupled case.

In the present paper we want to discuss the phenomenological properties of the heterotic M-theory. We also give details of the calculation leading to those results that were presented in [3]. This includes a presentation of the full effective four-dimensional $N=1$ supergravity action in leading and next-to-leading order, the mechanism of hidden sector gaugino condensation and its explicit consequences for supersymmetry breaking and the scalar potential and finally the resulting soft breaking terms in the 4-dimensional theory. We will at each step first explain the situation in the weakly coupled theory and then compare it to the results obtained in the M-theory case.

These results are obtained using the method of reduction and truncation that has been successfully applied to the weakly coupled case [8]. It is a simplified prescription that shows the main qualitative features of the effective $d=4$ effective theory. In orbifold compactification it would represent the fields and interactions in the untwisted sector. We compute Kähler potential $(K)$, superpotential $(W)$ and gauge kinetic function $(f)$ both in the weakly and strongly coupled regime and explain similarities and differences.

The results in leading order had been obtained previously [9, 10, 11, 12]. These papers mainly focused on the breakdown of supersymmetry via a Scherk-Schwarz mechanism. It remains to be seen, if and how such a mechanism can be related to the mechanism of gaugino condensation.

After our paper [3] appeared, several other groups also considered a discussion of the effective action beyond leading order as well as the mechanism of gaugino condensation [13, 14, 15, 16].

The paper will proceed as follows. In chapter 2 we discuss the scales and the question of unification as suggested in [2] and compare the two cases. In chapter 3 we review the old results in the weakly coupled heterotic string, including important corrections to the $f$-function at the one loop level. Chapter 4 deals with the effective $d=4$ action of M-theory using the method of reduction and truncation. In this case we have to deal with a nontrivial obstruction first encountered in [2]. It leads to an explicit $x^{11}$ dependence of certain fields, which is induced by vevs of antisymmetric tensor fields at the walls. To obtain the effective action in $d=4$ we have to integrate out this dependence. This then leads to corrections to $K$ and $f$ in next to leading order, which are very similar compared to those in the weakly coupled case. We also discuss the appearance and the size of a critical radius for $R_{11}$. The phenomenological fit presented in chapter 2 implies that we are not too far from that critical radius. In chapter 5 we discuss the mechanism of gaugino condensation. We start with the weakly coupled case and investigate the nature of the vev of the $H$-field (concerning some quantization conditions) and the cancellation of the vacuum energy. We then move to the strongly coupled case and see that such a cancellation appears locally at one wall. This supports the interpretation that the gaugino condensate is matched by a nontrivial vev of a Chern-Simons term. We then explicitly identify the mechanism of supersymmetry breakdown and the nature of the gravitino. The goldstino turns out to be the fermionic component of the $T$ superfield that represents essentially the radius 
of the 11th dimension. It is a bulk field, with a vev of its auxiliary component on one wall. Integrating out the 11 th dimension we then obtain explicitly the mass of the gravitino. Chapter 6 then deals with the induced soft breaking terms in the observable sector: scalar and gaugino masses. We point out a strong model dependence of the scalar masses and argue that they are not too different from the gravitino mass. This all is very similar to the situation in the weakly coupled case. We then compute the soft gaugino masses and see that in the strongly coupled case they are of the order of the gravitino mass. This comes from the fact that we are quite close to the critical radius and represents a decisive difference to the weakly coupled regime. In chapter 7 we discuss some immediate phenomenological consequences, give an outlook and mention some open questions.

\section{Scales and unification}

The framework of string theory might ultimately lead to an explanation of the unification of all fundamental coupling constants. In contrast to usual grand unified models describing exclusively gauge interactions we here have a unification with the gravitational interaction as well. One therefore expects the grand unified scale $M_{G U T}$ to be connected to the Planck scale.

\subsection{Weakly coupled $E_{8} \times E_{8}$ heterotic string}

Models of particle physics that are derived as the low energy limit of the $E_{8} \times E_{8}$ heterotic string are particularly attractive. They seem to be able to accommodate the correct gauge group and particle spectrum to lead to the supersymmetric extension to the $S U(3) \times S U(2) \times U(1)$ standard model. It is exactly in this framework that a unification of the gauge coupling constants is expected to appear at a scale $M_{G U T}=3 \cdot 10^{16}$ $\mathrm{GeV}$. This heterotic string theory (weakly coupled at the string scale) in fact gives a prediction for the relation between gauge and gravitational coupling constants. To see this explicitly let us have a look at the low energy effective action of the $d=10$ dimensional field theory:

$$
L=-\frac{4}{\left(\alpha^{\prime}\right)^{3}} \int d^{10} x \sqrt{g} \exp (-2 \phi)\left(\frac{1}{\left(\alpha^{\prime}\right)} R+\frac{1}{4} \operatorname{tr} F^{2}+\ldots\right),
$$

where $\alpha^{\prime}$ is the string tension and $\phi$ the dilaton field in $d=10$. A definite relation between gauge and gravitational coupling appears because of the universal behaviour of the dilaton term in eq. (1). The effective $d=4$-dimensional theory is obtained after compactification on a Calabi-Yau manifold with volume $V$ :

$$
L=-\frac{4}{\left(\alpha^{\prime}\right)^{3}} \int d^{4} x \sqrt{g} \exp (-2 \phi) V\left(\frac{1}{\left(\alpha^{\prime}\right)} R+\frac{1}{4} \operatorname{tr} F^{2}+\ldots\right) .
$$

Thus a universal factor $V \exp (-2 \phi)$ multiplies both the $R$ and $F^{2}$ terms. Newton's and Einstein's gravitational coupling constants are related as

$$
G_{N}=\frac{1}{8 \pi} \kappa_{4}^{2}=\frac{1}{M_{\text {Planck }}^{2}}
$$


with $M_{\text {Planck }} \approx 1.2 \cdot 10^{19} \mathrm{GeV}$. From eq. (2) we then deduce:

$$
G_{N}=\frac{\exp (2 \phi)\left(\alpha^{\prime}\right)^{4}}{64 \pi V}
$$

as well as

$$
\alpha_{G U T}=\frac{\exp (2 \phi)\left(\alpha^{\prime}\right)^{3}}{16 \pi V}
$$

leading to the relation

$$
G_{N}=\frac{\alpha_{G U T} \alpha^{\prime}}{4}
$$

Putting in the value for $M_{\text {Planck }}$ and $\alpha_{G U T} \approx 1 / 25$ one obtains a value for the string scale $M_{\text {string }}=\left(\alpha^{\prime}\right)^{-1 / 2}$ that is in the region of $10^{18} \mathrm{GeV}$. This is apparently much larger than the GUT-scale of $3 \cdot 10^{16} \mathrm{GeV}$, while naively one would like to identify $M_{\text {string }}$ with $M_{G U T}$. The discrepancy of the scales is sometimes called the unification problem in the framework of the weakly coupled heterotic string. Of course, the above argumentation is rather simple and more sophisticated (threshold) calculations are needed to settle this issue. In any case, the natural appearance of $M_{\text {string }} \sim M_{G U T}$ would have been desirable. Let us now see how the situation looks in the case of heterotic string theory at stronger coupling.

\section{$2.2 \quad E_{8} \times E_{8}$ M-theory}

The effective action of the strongly coupled $E_{8} \times E_{8}-M$-theory in the "downstairs" approach is given by [1] (we take into account the numerical corrections found in [17])

$$
\begin{aligned}
& L=\frac{1}{\kappa^{2}} \int_{M^{11}} d^{11} x \sqrt{g}[-\frac{1}{2} R-\frac{1}{2} \bar{\psi}_{I} \Gamma^{I J K} D_{J}\left(\frac{\Omega+\hat{\Omega}}{2}\right) \psi_{K}-\frac{1}{48} G_{I J K L} G^{I J K L} \\
&-\frac{\sqrt{2}}{384}\left(\bar{\psi}_{I} \Gamma^{I J K L M N} \psi_{N}+12 \bar{\psi}^{J} \Gamma^{K L} \psi^{M}\right)\left(G_{J K L M}+\hat{G}_{J K L M}\right) \\
&\left.-\frac{\sqrt{2}}{3456} \epsilon^{I_{1} I_{2} \ldots I_{11}} C_{I_{1} I_{2} I_{3}} G_{I_{4} \ldots I_{7}} G_{I_{8} \ldots I_{11}}\right] \\
&+\frac{1}{4 \pi\left(4 \pi \kappa^{2}\right)^{2 / 3}} \int_{M_{i}^{10}} d^{10} x \sqrt{g}\left[-\frac{1}{4} F_{i A B}^{a} F_{i}^{a A B}-\frac{1}{2} \bar{\chi}_{i}^{a} \Gamma^{A} D_{A}(\hat{\Omega}) \chi_{i}^{a}\right. \\
&\left.-\frac{1}{8} \bar{\psi}_{A} \Gamma^{B C} \Gamma^{A}\left(F_{i B C}^{a}+\hat{F}_{i B C}^{a}\right) \chi_{i}^{a}+\frac{\sqrt{2}}{48}\left(\bar{\chi}_{i}^{a} \Gamma^{A B C} \chi_{i}^{a}\right) \hat{G}_{A B C 11}\right]
\end{aligned}
$$

where $M^{11}$ is the "downstairs" manifold while $M_{i}^{10}$ are its 10 -dimensional boundaries. In the lowest approximation $M^{11}$ is just a product $M^{4} \times X^{6} \times S^{1} / Z_{2}$. Compactifying to $d=4$ in such an approximation we obtain [2, 17]

$$
\begin{gathered}
G_{N}=\frac{\kappa_{4}^{2}}{8 \pi}=\frac{\kappa^{2}}{8 \pi R_{11} V}, \\
\alpha_{G U T}=\frac{\left(4 \pi \kappa^{2}\right)^{2 / 3}}{V}
\end{gathered}
$$


with $V$ the volume of the Calabi-Yau manifold $X^{6}$ and $R_{11}=\pi \rho$ the $S^{1} / Z_{2}$ length.

The fundamental mass scale of the 11 -dimensional theory is given by $M_{11}=\kappa^{-2 / 9}$. Let us see which value of $M_{11}$ is favoured in a phenomenological application. For that purpose we identify the Calabi-Yau volume $V$ with the GUT-scale: $V \sim\left(M_{G U T}\right)^{-6}$. From (9) and the value of $\alpha_{G U T}=1 / 25$ at the grand unified scale, we can then deduce the value of $M_{11}$

$$
V^{1 / 6} M_{11}=(4 \pi)^{1 / 9} \alpha_{G U T}^{-1 / 6} \approx 2.3,
$$

to be a few times larger than the GUT-scale. In a next step we can now adjust the gravitational coupling constant by choosing the appropriate value of $R_{11}$ using (8). This leads to

$$
R_{11} M_{11}=\left(\frac{M_{\text {Planck }}}{M_{11}}\right)^{2} \frac{\alpha_{G U T}}{8 \pi(4 \pi)^{2 / 3}} \approx 2.9 \cdot 10^{-4}\left(\frac{M_{\text {Planck }}}{M_{11}}\right)^{2}
$$

This simple analysis tells us the following:

- In contrast to the weakly coupled case ( where we had a prediction (6)), the correct value of $M_{\text {Planck }}$ can be fitted by adjusting the value of $R_{11}$.

- The numerical value of $R_{11}^{-1}$ turns out to be approximately an order of magnitude smaller than $M_{11}$.

- Thus the 11th dimension appears to be larger than the dimensions compactified on the Calabi-Yau manifold, and at an intermediate stage the world appears 5-dimensional with two 4-dimensional boundaries (walls).

We thus have the following picture of the evolution and unification of coupling constants. At low energies the world is 4-dimensional and the couplings evolve accordingly with energy: a logarithmic variation of gauge coupling constants and the usual power law behaviour for the gravitational coupling. Around $R_{11}^{-1}$ we have an additional 5th dimension and the power law evolution of the gravitational interactions changes. Gauge couplings are not effected at that scale since the gauge fields live on the walls and do not feel the existence of the 5 th dimension. Finally at $M_{G U T}$ the theory becomes $11-$ dimensional and both gravitational and gauge couplings show a power law behaviour and meet at the scale $M_{11}$, the fundamental scale of the theory. It is obvious that the correct choice of $R_{11}$ is needed to achieve unification. We also see that, although the theory is weakly coupled at $M_{G U T}$, this is no longer true at $M_{11}$. The naive estimate for the evolution of the gauge coupling constants between $M_{G U T}$ and $M_{11}$ goes with the sixth power of the scale. At $M_{11}$ we thus expect unification of the couplings at $\alpha \sim O(1)$. In that sense, the $\mathrm{M}$-theoretic description of the heterotic string gives an interpolation between weak coupling and moderate coupling. In $d=4$ this is not strong-weak coupling duality in the usual sense. We shall later come back to these questions when we discuss the appearance of a critical limit on the size of $R_{11}$.

These are, of course, rather qualitative results. In order to get a more quantitative feeling for the range of $M_{11}$ and $R_{11}$, let us be a bit more specific and write the relation of the unification scale $M_{G U T}$ to the characteristic size of the Calabi-Yau space as:

$$
V^{1 / 6}=a M_{G U T}^{-1}
$$


In the spirit of our phenomenological ansatz we expect the parameter $a$ to be of order unity. Using the above identification and the value of $M_{G U T}=3 \cdot 10^{16} \mathrm{GeV}$ we obtain:

$$
M_{11} \approx \frac{2.3}{a} M_{G U T}
$$

As said before, the scale $M_{11}$ occurs to be of the order of the unification scale $M_{G U T}$. However, we do not expect $M_{11}$ to be smaller than $M_{G U T}$ because we need the ordinary logarithmic evolution of the gauge coupling constants up to $M_{G U T}$. In fact, $M_{11}$ should be somewhat bigger in order to allow for the evolution of $\alpha$ from its unification value $1 / 25$ to the strong regime. Thus, we obtain phenomenologically interesting solutions if the parameter $a$ is quite close to 1 . Putting the above value of $M_{11}$ into eq. (11) we get the length of $S^{1} / Z_{2}$ :

$$
R_{11} \approx 9.2 a^{2} M_{11}^{-1} \approx 4 a^{3} M_{G U T}^{-1} .
$$

It is about one order of magnitude bigger than the scale characteristic for the 11dimensional theory. This is the reason for the relatively large value of the $d=4$ Planck Mass. Of course $R_{11}$ can not be too large. For $a<2.3$ (values corresponding to $M_{11}>M_{G U T}$ ) we obtain $R_{11}^{-1}>6.2 \cdot 10^{14} \mathrm{GeV}$ (as we discussed, the parameter $a$ should not be too different from 1 which gives $R_{11}^{-1}$ close to $7.4 \cdot 10^{15} \mathrm{GeV}$ ). Smaller values of $R_{11}^{-1}$ seem to be very unnatural. Trying to push $R_{11}^{-1}$ to smaller values would need a redefinition of $M_{11}$. For that purpose in [9] a definition $m_{11}=2 \pi\left(4 \pi \kappa^{2}\right)^{-1 / 9}$ was used. This allows then to push $a$ to as high values as $2 \pi$. With these rather extreme choices of both $a$ and $m_{11}$ one would then be able to obtain $R_{11}^{-1}$ as small as $3 \cdot 10^{13} \mathrm{GeV}$. Values smaller than that (like values of $10^{12} \mathrm{GeV}$ as sometimes quoted in the literature) cannot be obtained. In any case, even values in the lower $10^{13} \mathrm{GeV}$ range seem to be in conflict with the critical value of $R_{11}$, as we shall see in chapter 4 .

\section{$3 \quad$ Weakly coupled heterotic string}

\subsection{The classical action}

We shall start from the $d=10$ effective field theory:

$$
L=-\frac{4}{\left(\alpha^{\prime}\right)^{3}} \int d^{10} x \sqrt{g} \exp (-2 \phi)\left(\frac{1}{\left(\alpha^{\prime}\right)} R+\frac{1}{4} \operatorname{tr} F^{2}+\frac{1}{12} \alpha^{\prime} H^{2}+\ldots\right),
$$

where we have included the three index tensor field strength

$$
H=d B+\omega^{Y M}-\omega^{L} .
$$

$B$ is the two-index antisymmetric tensor while

$$
\omega^{Y M}=\operatorname{Tr}\left(A F-\frac{2}{3} A^{3}\right)
$$

and

$$
\omega^{L}=\operatorname{Tr}\left(\omega R-\frac{2}{3} \omega^{3}\right)
$$


are the Yang-Mills and Lorentz-Chern-Simons terms, respectively. The addition of these terms in the definition of $H$ is needed for supersymmetry and anomaly freedom of the theory.

To obtain the effective theory in $d=4$ dimensions we use as an approximation the method of reduction and truncation explained in ref. [8]. It essentially corresponds to a torus compactification, while truncating states to arrive at a $d=4$ theory with $N=1$ supersymmetry. In string theory compactified on an orbifold this would describe the dynamics of the untwisted sector. We retain the usual moduli fields $S$ and $T$ as well as matter fields $C_{i}$ that transform nontrivially under the observable sector gauge group. In this approximation, the Kähler potential is given by [8, 6]

$$
G=-\log \left(S+S^{*}\right)-3 \log \left(T+T^{*}-2 C_{i}^{*} C_{i}\right)+\log |W|^{2}
$$

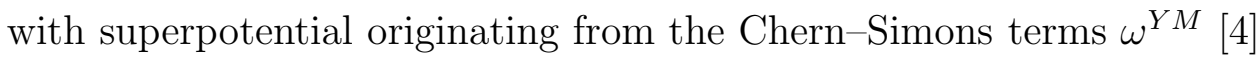

$$
W(C)=d_{i j k} C_{i} C_{j} C_{k}
$$

and the gauge kinetic function is given by the dilaton field

$$
f=S
$$

For a detailed discussion of this method and the explicit definition of the fields see the review [18]. These expressions for the $d=4$ effective action look quite simple and it remains to be seen whether this simplicity is true in general or whether it is an artifact of the approximation. Our experience with supergravity models tells us that the holomorphic functions $W$ and $f$ might be protected by nonrenormalization theorems, while the Kähler potential is strongly modified in perturbation theory. In addition we have to be aware of the fact that the expressions given above are at best representing a subsector of the theory. In orbifold compactification this would be the untwisted sector, and we know that the Kähler potential for twisted sectors fields will look quite different. Nonetheless the used approximation turned out to be useful for the discussion of those aspects of the theory that determine the dynamics of the $T$ and $S$-moduli. When trying to extract, however, detailed masses and other properties of the fields one should be aware of the fact, that some results might not be true in general and only appear as a result of the simplicity of the approximation.

\subsection{One-loop corrections}

Not much can be said about the details of the corrections to the Kähler potential. This has to be discussed on a model by model basis. The situation with the superpotential is quite easy. There we expect a nonrenormalization theorem to be at work. The inclusion of other sectors of the theory will lead to new terms in the superpotential that in general have $T$-dependent coefficients. Such terms can be computed in simple cases by using e.g. methods of conformal field theory [19].

The situation for $f$, the gauge-kinetic function is more interesting. Symmetries and holomorphicity lead us to believe, that although there are nontrivial corrections at one-loop, no more perturbative corrections are allowed at higher orders [20, 21]. The existence of such corrections at one loop seems to be intimately connected to the 
mechanism of anomaly cancellation in the $d=10$ theory [22, 23]. To see this consider one of the anomaly cancellation counter-terms introduced by Green and Schwarz [24]:

$$
\epsilon^{V O L K S W A G E N} B_{V O} \operatorname{Tr} F_{L K S W}^{2} F_{A G E N}^{2}
$$

We are interested in a $d=4$ theory with $N=1$ supersymmetry, and thus expect nontrivial vacuum expectation values for the curvature terms $\operatorname{Tr} R^{2}$ and field strengths $\operatorname{Tr} F^{2}$ in the extra six dimensions. Consistency of the theory requires a condition for the 3 -index tensor field strength. For $H$ to be well defined, the quantity

$$
d H=\operatorname{Tr} F^{2}-\operatorname{Tr} R^{2}
$$

has to vanish cohomologically [25]. In the simplest case (the so-called standard embedding leading to gauge group $E_{6} \times E_{8}$ ) one chooses equality pointwise $\operatorname{Tr} R^{2}=\operatorname{Tr} F^{2}$. Let us now assume that $\operatorname{Tr} F_{a g e n}^{2}$ is nonzero. The Green-Schwarz term given above by eq. (22) then leads to

$$
\epsilon^{m n} B_{m n} \epsilon^{\mu \nu \rho \sigma} \operatorname{Tr} F_{\mu \nu} F_{\rho \sigma}
$$

in the four-dimensional theory. An explicit inspection of the fields tells us that $\epsilon^{m n} B_{m n}$ is the pseudoscalar axion that belongs to the $T$-superfield. Upon supersymmetrization the term in eq. (24) will then correspond to a one-loop correction to the holomorphic $f$-function (21) that is proportional to $T$ with the coefficient fixed entirely by the anomaly considerations. This is, of course, nothing else than a threshold correction. In the simple case of the standard embedding with gauge group $E_{6} \times E_{8}$ one obtains e.g.

$$
f_{6}=S+\epsilon T ; \quad f_{8}=S-\epsilon T .
$$

respectively, where $\epsilon$ is the constant fixed by the anomaly. These results can be backed up by explicit calculations in string theory. In cases where such an explicit calculation is feasible, many more details about these corrections can be deduced. The above result (25) obtained in $d=10$ field theory represents an approximation of the exact result in the large $T$-limit. For a detailed discussion of these calculations and the limiting procedure see [26]. We have here mainly concentrated on that limit, because it represents a rather model independent statement.

Thus we have seen that there are corrections to the gauge-kinetic function at one loop. Their existence is found to be intimately related to the mechanism of anomaly cancellation. The corrections found are exactly those that are expected by general symmetry considerations [21]. In (25) we have given the result for the standard embedding. Coefficients might vary for more general cases, but the fact that they have opposite sign for the two separate groups is true in all known cases.

\subsection{Beyond one loop}

Not much can be said about the Kähler potential beyond one loop. Superpotential and $f$-function, however, should not receive further perturbative corrections. This implies that the knowledge of $f$ at one loop represents the full perturbative result. Combined with the fact that the coefficients are fixed by anomaly considerations one would then expect that this result for the $f$-function might be valid even beyond the weakly coupled limit. 


\section{Heterotic M-Theory}

Let us now review the calculation of [3]. In the strongly coupled case we perform a compactification from $d=11$ to $d=4$ using the method of reduction and truncation as above. For the metric we write

$$
g_{M N}^{(11)}=\left(\begin{array}{ccc}
c_{4} e^{-\gamma} e^{-2 \sigma} g_{\mu \nu} & & \\
& e^{\sigma} g_{m n} & \\
& & e^{2 \gamma} e^{-2 \sigma}
\end{array}\right)
$$

with $M, N=1 \ldots 11 ; \mu, \nu=1 \ldots 4 ; m, n=5 \ldots 10$ and $\operatorname{det}\left(g_{m n}\right)=1$. This is the frame in which the 11-dimensional Einstein action gives the ordinary Einstein action after the reduction do $d=4$ :

$$
-\frac{1}{2 \kappa^{2}} \int d^{11} x \sqrt{g^{(11)}} R^{(11)}=-\frac{c_{4} \hat{V}_{7}}{2 \kappa^{2}} \int d^{4} x \sqrt{g} R+\ldots
$$

where $\hat{V}_{7}=\int d^{7} x$ is the coordinate volume of the compact 7 -manifold and the scaling factor $c_{4}$ describes our freedom to choose the units in $d=4$. The most popular choice in the literature is $c_{4}=1$. This however corresponds to the unphysical situation in which the 4 -dimensional Planck mass is determined by the choice of $\hat{V}_{7}$ which is just a convention. With $c_{4}=1$ one needs further rescaling of the 4 -dimensional metric. We instead prefer the choice

$$
c_{4}=V_{7} / \hat{V}_{7}
$$

where $V_{7}=\int d^{7} x \sqrt{g^{(7)}}$ is the physical volume of the compact 7 -manifold. This way we recover eq. (8) in which the 4-dimensional Planck mass depends on the physical, and not coordinate, volume of the manifold on which we compactify. As a result, if we start from the product of the 4-dimensional Minkowski space and some 7-dimensional compact space (in the leading order of the expansion in $\kappa^{2 / 3}$ ) as a ground state in $d=11$ we obtain the Minkowski space with the standard normalization as the vacuum in $d=4$.

To find a more explicit formula for $c_{4}$ we have to discuss the fields $\sigma$ and $\gamma$ in some detail. In the leading approximation $\sigma$ is the overall modulus of the Calabi-Yau 6manifold. We can divide it into a sum of the vacuum expectation value, $\langle\sigma\rangle$, and the fluctuation $\tilde{\sigma}$. In general both parts could depend on all 11 coordinates but in practice we have to impose some restrictions. The vacuum expectation value can not depend on $x^{\mu}$ if the 4-dimensional theory is to be Lorentz-invariant. In the fluctuations we drop the dependence on the compact coordinates corresponding to the higher Kaluza-Klein modes. Furthermore, we know that in the leading approximation $\langle\sigma\rangle$ is just a constant, $\sigma_{0}$, while corrections depending on the internal coordinates, $\sigma_{1}$, are of the next order in $\kappa^{2 / 3}$. Thus, we obtain

$$
\sigma\left(x^{\mu}, x^{m}, x^{11}\right)=\langle\sigma\rangle\left(x^{m}, x^{11}\right)+\tilde{\sigma}\left(x^{\mu}\right)=\sigma_{0}+\sigma_{1}\left(x^{m}, x^{11}\right)+\tilde{\sigma}\left(x^{\mu}\right) .
$$

To make the above decomposition unique we define $\sigma_{0}$ by requiring that the integral of $\sigma_{1}$ over the internal space vanishes. The analogous decomposition can be also done for $\gamma$. With the above definitions the physical volume of the compact space is

$$
V_{7}=\int d^{7} x\left\langle e^{2 \sigma} e^{\gamma}\right\rangle=e^{2 \sigma_{0}} e^{\gamma_{0}} \hat{V}_{7}
$$


up to corrections of order $\kappa^{4 / 3}$. Thus, the parameter $c_{4}$ can be written as

$$
c_{4}=e^{2 \sigma_{0}} e^{\gamma_{0}} .
$$

The choice of the coordinate volumes is just a convention. For example in the case of the Calabi-Yau 6-manifold only the product $e^{3 \sigma} \hat{V}_{6}$ has physical meaning. For definiteness we will use the convention that the coordinate volumes are equal 1 in $M_{11}$ units. Thus, $\left\langle e^{3 \sigma}\right\rangle$ describes the Calabi-Yau volume in these units. Using eqs. (10,11) we obtain $e^{3 \sigma_{0}}=V M_{11}^{6} \approx(2.3)^{6}, e^{\gamma_{0}} e^{-\sigma_{0}}=R_{11} M_{11} \approx 9.2 a^{2}$. The parameter $c_{4}$ is equal to the square of the 4 -dimensional Planck mass in these units and numerically $c_{4} \approx(35 a)^{2}$.

\subsection{Leading order}

At the classical level we compactify on $M^{4} \times X^{6} \times S^{1} / Z_{2}$. This means that the vacuum expectation values $\langle\sigma\rangle$ and $\langle\gamma\rangle$ are just constants and eq. (29) reduces to

$$
\sigma=\sigma_{0}+\tilde{\sigma}\left(x^{\mu}\right), \quad \gamma=\gamma_{0}+\tilde{\gamma}\left(x^{\mu}\right)
$$

In such a situation $\sigma$ and $\gamma$ are 4-dimensional fields. We introduce two other 4dimensional fields by the relations

$$
\begin{aligned}
\frac{1}{4 ! c_{4}} e^{6 \sigma} G_{11 \lambda \mu \nu} & =\epsilon_{\lambda \mu \nu \rho}\left(\partial^{\rho} D\right) \\
C_{11 a \bar{b}} & =C_{11} \delta_{a \bar{b}}
\end{aligned}
$$

where $x^{a}\left(x^{\bar{b}}\right)$ is the holomorphic (antiholomorphic) coordinate of the Calabi-Yau manifold. Now we can define the dilaton and the modulus fields by

$$
\begin{aligned}
\mathcal{S} & =\frac{1}{(4 \pi)^{2 / 3}}\left(e^{3 \sigma}+i 24 \sqrt{2} D\right) \\
\mathcal{T} & =\frac{1}{(4 \pi)^{2 / 3}}\left(e^{\gamma}+i 6 \sqrt{2} C_{11}+C_{i}^{*} C_{i}\right)
\end{aligned}
$$

where the observable sector matter fields $C_{i}$ originate from the gauge fields $A_{M}$ on the 10-dimensional observable wall (and $M$ is an index in the compactified six dimensions). The Kähler potential takes its standard form as in eq. (19)

$$
K=-\log \left(\mathcal{S}+\mathcal{S}^{*}\right)-3 \log \left(\mathcal{T}+\mathcal{T}^{*}-2 C_{i}^{*} C_{i}\right)
$$

The imaginary part of $\mathcal{S}(\operatorname{Im} \mathcal{S})$ corresponds to the model independent axion, and with the above normalization the gauge kinetic function is $f=\mathcal{S}$. We have also

$$
W(C)=d_{i j k} C_{i} C_{j} C_{k}
$$

This is very similar to the weakly coupled case. 


\subsection{Next to leading order}

Before drawing any conclusion from the formulae obtained above we have to discuss a possible obstruction at the next to leading order. For the 3 -index tensor field $H$ in $d=10$ supergravity to be well defined one has to satisfy $d H=\operatorname{tr} F_{1}^{2}+\operatorname{tr} F_{2}^{2}-\operatorname{tr} R^{2}=0$ cohomologically. In the simplest case of the standard embedding one assumes $\operatorname{tr} F_{1}^{2}=$ $\operatorname{tr} R^{2}$ locally and the gauge group is broken to $E_{6} \times E_{8}$. Since in the M-theory case the two different gauge groups live on the two different boundaries (walls) of spacetime such a cancellation point by point is no longer possible [2]. We expect nontrivial vacuum expectation values (vevs) of

$$
(d G) \propto \sum_{i} \delta\left(x^{11}-x_{i}^{11}\right)\left(\operatorname{tr} F_{i}^{2}-\frac{1}{2} \operatorname{tr} R^{2}\right)
$$

at least on one boundary ( $x_{i}^{11}$ is the position of $i$-th boundary). In the case of the standard embedding we would have $\operatorname{tr} F_{1}^{2}-\frac{1}{2} \operatorname{tr} R^{2}=\frac{1}{2} \operatorname{tr} R^{2}$ on one and $\operatorname{tr} F_{2}^{2}-\frac{1}{2} \operatorname{tr} R^{2}=$ $-\frac{1}{2} \operatorname{tr} R^{2}$ on the other boundary. This might pose a severe problem since a nontrivial vev of $G$ might be in conflict with supersymmetry $\left(G_{11 A B C}=H_{A B C}\right)$. The supersymmetry transformation law in $d=11$ reads

$$
\delta \psi_{M}=D_{M} \eta+\frac{\sqrt{2}}{288} G_{I J K L}\left(\Gamma_{M}^{I J K L}-8 \delta_{M}^{I} \Gamma^{J K L}\right) \eta+\ldots
$$

Supersymmetry will be broken unless e.g. the derivative term $D_{M} \eta$ compensates the nontrivial vev of $G$. Witten has shown [2] that such a cancellation can occur and constructed the solution in the linearized approximation (linear in the expansion parameter $\left.\kappa^{2 / 3}\right)$ 円. This solution requires some modification of the metric on $M^{11}$ :

$$
g_{M N}^{(11)}=\left(\begin{array}{ccc}
(1+b) \eta_{\mu \nu} & & \\
& \left(g_{i j}+h_{i j}\right) & \\
& & \left(1+\gamma^{\prime}\right)
\end{array}\right) .
$$

$M^{11}$ is no longer a direct product $M^{4} \times X^{6} \times S^{1} / Z_{2}$ because $b, h_{i j}$ and $\gamma^{\prime}$ depend now on the compactified coordinates. The volume of $X^{6}$ depends on $x^{11}$ [2]:

$$
\frac{\partial}{\partial x^{11}} V=-\frac{\sqrt{2}}{8} \int d^{6} x \sqrt{g} \omega^{A B} \omega^{C D} G_{A B C D}
$$

where the integral is over the Calabi-Yau manifold $X^{6}$ and $\omega$ is the corresponding Kähler form. The parameter $(1+b)$ is the scale factor of the Minkowski 4-manifold and depends on $x^{11}$ in the following way

$$
\frac{\partial}{\partial x^{11}} b=\frac{1}{2} \frac{\partial}{\partial x^{11}} \log v_{4}=\frac{\sqrt{2}}{24} \omega^{A B} \omega^{C D} G_{A B C D}
$$

where $v_{4}$ is the physical volume for some fixed coordinate volume in $M^{4}$. In our simple reduction and truncation method with the metric $g_{M N}^{(11)}$ given by eq. (26) we can reproduce the $x^{11}$ dependence of $V$ and $v_{4}$. The volume of $X^{6}$ is determined by $\sigma$ :

$$
\frac{\partial}{\partial x^{11}} \log V=\frac{\partial}{\partial x^{11}}(3\langle\sigma\rangle)=3 \frac{\partial}{\partial x^{11}} \sigma
$$

\footnotetext{
${ }^{1}$ For a discussion beyond this approximation in the weakly coupled case see ref. [26].
} 
while the scale factor of $M^{4}$ can be similarly expressed in terms of $\sigma$ and $\gamma$ fields:

$$
\frac{\partial}{\partial x^{11}} \log v_{4}=-\frac{\partial}{\partial x^{11}}(2\langle\gamma\rangle+4\langle\sigma\rangle)=-\frac{\partial}{\partial x^{11}}(2 \gamma+4 \sigma) \text {. }
$$

Substituting $\langle\sigma\rangle$ with $\sigma$ in the above two equations is allowed because, due to our decomposition (29), only the vev of $\sigma$ depends on the internal coordinates (the same is true for $\gamma$ ). The scale factor $b$ calculated in ref. [2] depends also on the Calabi-Yau coordinates. Such a dependence can not be reproduced in our simple reduction and truncation compactification so we have to average eq. (43) over $X^{6}$. Using equations (42 45) after such an averaging we obtain (to leading order in the expansion parameter $\left.\kappa^{2 / 3}\right)$ [3

$$
\frac{\partial \gamma}{\partial x^{11}}=-\frac{\partial \sigma}{\partial x^{11}}=\frac{\sqrt{2}}{24} \frac{\int d^{6} x \sqrt{g} \omega^{A B} \omega^{C D} G_{A B C D}}{\int d^{6} x \sqrt{g}} .
$$

Substituting the vacuum expectation value of $G$ found in [2] we can rewrite it in the form

$$
\frac{\partial \gamma}{\partial x^{11}}=-\frac{\partial \sigma}{\partial x^{11}}=\frac{2}{3} \alpha \kappa^{2 / 3} V^{-2 / 3}
$$

where

$$
\alpha=\frac{\pi c}{2(4 \pi)^{2 / 3}}
$$

and $\mathrm{c}$ is a constant of order unity given for the standard embedding of the spin connection by

$$
c=V^{-1 / 3}\left|\int \frac{\omega \wedge \operatorname{tr}(R \wedge R)}{8 \pi^{2}}\right| .
$$

Our calculations, as those of Witten, are valid only in the leading nontrivial order in the $\kappa^{2 / 3}$ expansion. The expression (47) for the derivatives of $\sigma$ and $\gamma$ have explicit factor $\kappa^{2 / 3}$. This means that we should take the lowest order value for the Calabi-Yau volume in that expression. An analogous procedure has been used in obtaining all formulae presented in this paper. We always expand in $\kappa^{2 / 3}$ and drop all terms which are of higher order than our approximation. Taking the above into account and using our units in which $M_{11}=1$ we can rewrite eq. (47) in the simple form:

$$
\frac{\partial \gamma}{\partial x^{11}}=-\frac{\partial \sigma}{\partial x^{11}}=\frac{2}{3} \alpha e^{-2 \sigma_{0}}
$$

Eqs. (46 50) represent one of the main results of ref. [3]. As we will see in the following, this result contains all the information to deduce the effective action, i.e. Kähler potential, superpotential and gauge kinetic function of the 4 -dimensional effective supergravity theory.

As we showed in [3], it is the above dependence of $\sigma$ and $\gamma$ on $x^{11}$ that leads to these consequences. One has to be careful in defining the fields in $d=4$. It is obvious, that the 4 -dimensional fields $\mathcal{S}$ and $\mathcal{T}$ can not be any longer defined by eqs. (35, 36) because now $\sigma$ and $\gamma$ are 5-dimensional fields. We have to integrate out the dependence on the 11th coordinate. In the present approximation, this procedure is quite simple: we have to replace $\sigma$ and $\gamma$ in the definitions of $\mathcal{S}$ and $\mathcal{T}$ with their averages over the $S^{1} / Z_{2}$ 
interval [3]. With the linear dependence of $\sigma$ and $\gamma$ on $x^{11}$ their average values coincide with the values taken at the middle of the $S^{1} / Z_{2}$ interval

$$
\begin{aligned}
& \bar{\sigma}=\sigma\left(\frac{\pi \rho}{2}\right)=\sigma_{0}+\tilde{\sigma}\left(x^{\mu}\right), \\
& \bar{\gamma}=\gamma\left(\frac{\pi \rho}{2}\right)=\gamma_{0}+\tilde{\gamma}\left(x^{\mu}\right) .
\end{aligned}
$$

When we reduce the boundary part of the Lagrangian of M-theory to 4 dimensions we find exponents of $\sigma$ and $\gamma$ fields evaluated at the boundaries. Using eqs. (29) and (50) we get

$$
\begin{aligned}
& \left.e^{-\gamma}\right|_{M_{i}^{10}}=e^{-\gamma_{0}} \pm \frac{1}{3} \alpha e^{-3 \sigma_{0}} \\
& \left.e^{3 \sigma}\right|_{M_{i}^{10}}=e^{3 \sigma_{0}} \pm \alpha e^{\gamma_{0}}
\end{aligned}
$$

The above formulae have very important consequences for the definitions of the Kähler potential and the gauge kinetic functions. For example, the coefficient in front of the $D_{\mu} C_{i}^{*} D^{\mu} C_{i}$ kinetic term is proportional to $e^{-\gamma}$ evaluated at the $E_{6}$ wall where the matter fields propagate. At the lowest order this was just $e^{-\gamma_{0}}$ or $\langle\mathcal{T}\rangle^{-1}$ up to some numerical factor. From eq. (53) we see that at the next to leading order also $\langle\mathcal{S}\rangle^{-1}$ is involved with relative coefficient $\alpha / 3$. Taking such corrections into account we find that at this order the Kähler potential is given by

$$
K=-\log \left(\mathcal{S}+\mathcal{S}^{*}\right)+\frac{2 \alpha C_{i}^{*} C_{i}}{\mathcal{S}+\mathcal{S}^{*}}-3 \log \left(\mathcal{T}+\mathcal{T}^{*}-2 C_{i}^{*} C_{i}\right)
$$

with $\mathcal{S}$ and $\mathcal{T}$ now defined by

$$
\begin{aligned}
\mathcal{S} & =\frac{1}{(4 \pi)^{2 / 3}}\left(e^{3 \bar{\sigma}}+i 24 \sqrt{2} \bar{D}+\alpha C_{i}^{*} C_{i}\right), \\
\mathcal{T} & =\frac{1}{(4 \pi)^{2 / 3}}\left(e^{\bar{\gamma}}+i 6 \sqrt{2} \bar{C}_{11}+C_{i}^{*} C_{i}\right)
\end{aligned}
$$

where bars denote averaging over the 11th dimension. It might be of some interest to note that the combination $\langle\mathcal{S}\rangle\langle\mathcal{T}\rangle^{3}$ is independent of $x^{11}$ even before this averaging procedure took place.

Equipped with this definition the calculation of the gauge kinetic function(s) from eqs. (50, 54) becomes a trivial exercise [3]. In the five-dimensional theory $f$ depends on the 11-dimensional coordinate as well, thus the gauge kinetic function takes different values at the two walls. The averaging procedure allows us to deduce these functions directly. For the simple case at hand (the so-called standard embedding) eq. (54) gives [3]

$$
f_{6}=\mathcal{S}+\alpha \mathcal{T} ; \quad f_{8}=\mathcal{S}-\alpha \mathcal{T} .
$$

It is a special property of the standard embedding that the coefficients are equal and opposite. The coefficients vary for more general cases. This completes the discussion of

${ }^{2}$ Our solution is valid only for terms at most linear in $\alpha$. Keeping this in mind we could write the Kähler potential also in the form $K=-\log \left(\mathcal{S}+\mathcal{S}^{*}-2 \alpha C_{i}^{*} C_{i}\right)-3 \log \left(\mathcal{T}+\mathcal{T}^{*}-2 C_{i}^{*} C_{i}\right)$. 
the $d=4$ effective action in next to leading order, noting that the superpotential does not receive corrections at this level.

The nontrivial dependence of $\sigma$ and $\gamma$ on $x^{11}$ can also enter definitions and/or interactions of other 4-dimensional fields. Let us next consider the gravitino. After all we have to show that this field is massless to give the final proof that the given solution respects supersymmetry. Its 11 -dimensional kinetic term

$$
-\frac{1}{2} \sqrt{g} \bar{\psi}_{I} \Gamma^{I J K} D_{J} \psi_{K}
$$

remains diagonal after compactification to $d=4$ if we define the 4 -dimensional gravitino, $\psi_{\mu}^{(4)}$, and dilatino,$\psi_{11}^{(4)}$, fields by the relations

$$
\begin{aligned}
\psi_{\mu} & =e^{-\left(\sigma-\sigma_{0}\right) / 2} e^{-\left(\gamma-\gamma_{0}\right) / 4}\left(\psi_{\mu}^{(4)}+\frac{1}{\sqrt{6}} \Gamma_{\mu} \psi_{11}^{(4)}\right) \\
\psi_{11} & =-\frac{2}{\sqrt{6}} e^{\left(\sigma-\sigma_{0}\right) / 2} e^{\left(\gamma-\gamma_{0}\right) / 4} \Gamma^{11} \psi_{11}^{(4)}
\end{aligned}
$$

The $d=11$ kinetic term (59) gives after the compactification also a mass term for the $d=4$ gravitino of the form

$$
\frac{3}{8} e^{\sigma_{0}} e^{-\gamma_{0}} \frac{\partial \gamma}{\partial x^{11}}=\frac{\sqrt{2}}{64} e^{\sigma_{0}} e^{-\gamma_{0}} \frac{\int d^{6} x \sqrt{g} \omega^{A B} \omega^{C D} G_{A B C D}}{\int d^{6} x \sqrt{g}}=\frac{1}{4} \alpha e^{-\sigma_{0}} e^{-\gamma_{0}} .
$$

The sources of such a term are nonzero values of the spin connection components $\omega_{\mu}^{\alpha 11}$ and $\omega_{m}^{a 11}$ resulting from the $x^{11}$ dependence of the metric. It is a constant mass term from the 4-dimensional point of view. This, however, does not mean that the gravitino mass is nonzero. There is another contribution from the 11-dimensional term

$$
-\frac{\sqrt{2}}{384} \sqrt{g} \bar{\psi}_{I} \Gamma^{I J K L M N} \psi_{N}\left(G_{J K L M}+\hat{G}_{J K L M}\right)
$$

After redefining fields according to (60,61) and averaging the nontrivial vacuum expectation value of $G$ over $X^{6}$ we get from eq. (63) a mass term which exactly cancels the previous contribution (62). The gravitino is massless - the result which we expect in a model with unbroken supersymmetry and vanishing cosmological constant. Thus, we find that our simple reduction and truncation method (including the correct $x^{11}$ dependence in next to leading order) reproduces the main features of the model.

\subsection{Critical radius}

The factor $\langle\exp (3 \sigma)\rangle$ represents the volume of the six-dimensional compact space in units of $M_{11}^{-6}$. The $x^{11}$ dependence of $\sigma$ then leads to the geometrical picture that the volume of this space varies with $x^{11}$ and differs at the two boundaries:

$$
V_{E_{8}}=V_{E_{6}}-2 \pi^{2} \rho\left(\frac{\kappa}{4 \pi}\right)^{2 / 3}\left|\int \omega \wedge \frac{\operatorname{tr}(F \wedge F)-\frac{1}{2} \operatorname{tr}(R \wedge R)}{8 \pi^{2}}\right|
$$

where the integral is over $X^{6}$ at the $E_{6}$ boundary. In the given approximation, this variation is linear, and for growing $\rho$ the volume on the $E_{8}$ side becomes smaller and 
smaller. At a critical value of $\rho$ the volume will thus vanish and this will provide us with an upper limit on $\rho$ :

$$
\rho<\rho_{\text {crit }}=\frac{(4 \pi)^{2 / 3}}{c \pi^{2}} M_{11}^{3} V_{E_{6}}^{2 / 3}
$$

where $c$ was defined in eq. (49). To estimate the numerical value of $\rho_{\text {crit }}$ we first recall that from eq. (8) we obtained

$$
M_{11} V_{E_{6}}^{1 / 6}=\left(\alpha_{G U T}(4 \pi)^{-2 / 3}\right)^{-1 / 6} \approx 2.3 .
$$

Thus, we get

$$
\rho^{-1}>\rho_{\text {crit }}^{-1} \approx 0.16 c V_{E_{6}}^{-1 / 6} .
$$

The numerical value of $V$ at the $E_{6}$ boundary depends on what we identify with the unification scale $M_{G U T}$ via eq. (12):

$$
V_{E_{6}}^{-1 / 6}=a M_{G U T}^{-1}
$$

with $a$ somewhere between 1 and about 2 . Thus, the bound (67) can be written in the form

$$
R_{11}^{-1}>0.05 \frac{c}{a} M_{G U T}
$$

For the phenomenological applications we have to check whether our preferred choice of $6.2 \cdot 10^{14} \mathrm{GeV}<R_{11}^{-1}<7.4 \cdot 10^{15} \mathrm{GeV}$ that fits the correct value of the $d=4$ Planck mass satisfies the bound (69). In a rather extreme case of $c=1$ and $a=2.3$ we find that the upper bound on $R_{11}^{-1}$ is of the order of $6.5 \cdot 10^{14} \mathrm{GeV}$. Even for $c=1$ this bound goes up to about $1.5 \cdot 10^{15} \mathrm{GeV}$ if we identify $V^{-1 / 6}$ with $M_{G U T}$. Although some coefficients are model dependent we find in general that the bound can be satisfied, but that $R_{11}$ is quite close to its critical value. Values of $R_{11}^{-1}$ about $10^{12} \mathrm{GeV}$ as necessary in [9] seem to be beyond the critical value, even with the modifications discussed at the end of Chapter 2. In any case, models where supersymmetry is broken by a Scherk-Schwarz mechanism seem to require the absence of the next to leading order corrections in (58), i.e. $\alpha=0$. It remains to be seen whether such a possibility can be realized.

\subsection{Connection to the weakly coupled case}

Inspection of (25) and (58) reveals a close connection between the strongly and weakly coupled case [27, 26]. The variation of the Calabi-Yau manifold volume as discussed above is the analogue of the one loop correction of the gauge kinetic function (25) in the weakly coupled case and has the same origin, namely a Green-Schwarz anomaly cancellation counterterm. In fact, also in the strongly coupled case this leads to a correction for the gauge coupling constants at the $E_{6}$ and $E_{8}$ side. As seen, gauge couplings are no longer given by the (averaged) $\mathcal{S}$-field, but by that combination of

\footnotetext{
${ }^{3}$ With $V$ depending on $x^{11}$ we have to specify which values should be used in eqs. (8,9, 12, . The appropriate choice in the expression for $G_{N}$ is the average value of $V$ while in the expressions for $\alpha_{G U T}$ and for the $V-M_{G U T}$ relation we have to use $V$ evaluated at the $E_{6}$ wall.
} 
the (averaged) $\mathcal{S}$ and $\mathcal{T}$ fields which corresponds to the $\mathcal{S}$-field before averaging at the given boundary leading to

$$
f_{6,8}=\mathcal{S} \pm \alpha \mathcal{T}
$$

at the $E_{6}\left(E_{8}\right)$ side respectively. The critical value of $R_{11}$ will correspond to infinitely strong coupling at the $E_{8}$ side $\mathcal{S}-\alpha \mathcal{T}=0$. Since we are here close to criticality a correct phenomenological fit of $\alpha_{\mathrm{GUT}}=1 / 25$ should include this correction $\alpha_{\mathrm{GUT}}^{-1}=\mathcal{S}+\alpha \mathcal{T}$ where $\mathcal{S}$ and $\alpha \mathcal{T}$ give comparable contributions. This is a difference to the weakly coupled case, where in $f=S+\epsilon T$ the latter contribution was small compared to $S$. This stable result for the corrections to $f$ when going from weak coupling to strong coupling is only possible because of the rather special properties of $f$. $f$ does not receive further perturbative corrections beyond one loop [20, 21], and the one loop corrections are determined by the anomaly considerations. The formal expressions for the corrections are identical, the difference being only that in the strongly coupled case these corrections are as important as the classical value.

\section{$5 \quad$ Supersymmetry breaking at the hidden wall}

We shall now discuss the question of supersymmetry breakdown within this framework. We consider the breakdown of supersymmetry in a hidden sector, transmitted to the observable sector via gravitational interactions. Such a scenario was suggested in [28] after having observed that gaugino condensation can break supersymmetry in $d=4$ supergravity models. A nontrivial gauge kinetic function $f$ seems to be necessary for such a mechanism to work [29]. In the heterotic string both ingredients, a hidden sector $E_{8}$ and a nontrivial $f$, were present in a natural way and a coherent picture of supersymmetry breakdown via gaugino condensation emerged [4, 5, 6]. In the strongly coupled case, such a mechanism can be realized as well [7, 3]. In fact the notion of the hidden sector acquires a geometrical interpretation: the gaugino condensate forms at one boundary (the hidden wall) of spacetime. We shall now discuss this mechanism in detail. First we review the situation in the weakly coupled case. Our aim then is to compare the strong coupling regime with the weak coupling regime and clarify similarities as well as differences.

\section{$5.1 \quad$ Weak coupling case}

Let us first discuss supersymmetry breaking in the weakly coupled case using the action of $d=10$ supergravity. Supersymmetry transformation laws for the $d=10$ gravitino fields $\psi_{M}$ and the dilatino field $\lambda$ are written ${ }^{4}$

$$
\begin{aligned}
\delta \lambda & =\frac{1}{8} \varphi^{-3 / 4} \Gamma^{M N P} H_{M N P}+\frac{\sqrt{2}}{384} \Gamma^{M N P} \bar{\chi}^{a} \Gamma_{M N P} \chi^{a}+\ldots, \\
\delta \psi_{M} & =\frac{\sqrt{2}}{32} \varphi^{-3 / 4}\left(\Gamma_{M}^{N P Q}-9 \delta_{M}^{N} \Gamma^{P Q}\right) H_{N P Q}
\end{aligned}
$$

\footnotetext{
${ }^{4}$ Here we use the conventions of [30], where the Lagrangian is given in the Einstein frame. To recover the effective action (15) in the string frame, one has to make a proper Weyl transformation and identify $\varphi=\exp (\phi / 3)$.
} 


$$
+\frac{1}{256}\left(\Gamma_{M N P Q}-5 g_{M N} \Gamma_{P Q}\right) \bar{\chi}^{a} \Gamma^{N P Q} \chi^{a}+\ldots,
$$

implying that a condensate of gauginos $\bar{\chi} \chi$ and/or non-vanishing vevs of the $H$ fields may break supersymmetry. Here we assume the appearance of the gaugino condensate in the hidden sector

$$
\left\langle\bar{\chi}^{a} \Gamma_{m n p} \chi^{a}\right\rangle=\Lambda^{3} \epsilon_{m n p},
$$

with $\Lambda$ being the gaugino condensation scale and $\epsilon_{m n p}$ the covariantly constant holomorphic three-form. The perfect square structure seen in the Lagrangian [5]

$$
-\frac{3}{4} \varphi^{-3 / 2}\left(H_{M N P}-\sqrt{2} \varphi^{3 / 4} \bar{\chi}^{a} \Gamma_{M N P} \chi^{a}\right)^{2}
$$

will be a very important ingredient to discuss the quantitative properties of the mechanism. When reducing to the $d=4$ effective action we will find a cancellation of the vevs of the $H$ field and the gaugino condensate at the minimum of the potential such that the term in eq. (73) vanishes. Before we look at this in detail, let us first comment on such a possible vev of $H$ and a possible quantization condition of the antisymmetric tensor. In [31] it was shown, that an antisymmetric tensor field $H=d B$ has a quantized vacuum expectation value. In many subsequent papers this has been incorrectly taken as an argument for the quantization of the vev of $H=d B+\omega^{Y M}-\omega^{L}$ as given in eq. (16). The correct way to interpret this situation is to have a cancellation of the gaugino condensate with the vev of a Chern-Simons term [6], for which such a quantization condition does not hold. After all the Chern-Simons term $\omega^{Y M}$ contains the superpotential of the $d=4$ effective theory [4]. This cancellation leads to a certain combination of $\psi_{M}$ and $\lambda$ as the candidate goldstino that will provide the longitudinal component of the gravitino. While in $d=10$ this looks rather complicated, it simplifies tremendously once one reduces to $d=4$. Qualitatively the scalar potential takes the following form at the classical level (for the detailed factors see [32]):

$$
V=\frac{1}{S T^{3}}\left[\left|W-2(S T)^{3 / 2}(\bar{\chi} \chi)\right|^{2}+\frac{T}{3}\left|\frac{\partial W}{\partial C}\right|^{2}\right] .
$$

We observe the important fact that the potential is positive and vanishes at the minimum. Thus we have broken supersymmetry with a vanishing cosmological constant at the classical level. The first term in the brackets of eq. (74) corresponds to the contribution from eq. (73) once reduced to $d=4$ and vanishes at the minimum. In the $d=4$ theory it represents the auxiliary component $F_{S}$ of the dilaton superfield $S$. Thus we have $F_{S}=0$ and supersymmetry is broken by a nonvanishing vev of $F_{T}$ [6]. The goldstino is then the fermion in the $T$-multiplet and we are dealing with a situation that has later been named moduli-dominated supersymmetry breakdown. This fact has its origin in the special properties of the $d=10$ action (the term in eq. (73)) and seems to be of rather general validity. The statement $F_{S}=0$ is, of course, strictly valid only in the classical theory. The corrections discussed in section 3, eq. (25) will slightly change these results as we shall discuss in section 6 .

Having minimized the potential and identified the goldstino we can now compute the gravitino mass according to the standard procedure. The result has a direct physical meaning because we are dealing with a theory with vanishing vacuum energy. We obtain

$$
m_{3 / 2} \sim \frac{F_{T}}{M_{\text {Planck }}} \sim \frac{\Lambda^{3}}{M_{\text {Planck }}^{2}} .
$$


A value of $\Lambda \sim 10^{13} \mathrm{GeV}$ will thus lead to a gravitino mass in the $\mathrm{TeV}$ region. So far is our review of the mechanism of gaugino condensation in the weakly coupled theory.

\subsection{Strongly coupled case}

Next we turn to supersymmetry breaking in the strongly coupled case $(d=11$ M-theory

picture) and start with the $d=11$ action. Supersymmetry transformation laws for the gravitino fields in this case are given by

$$
\begin{aligned}
\delta \psi_{A}= & D_{A} \eta+\frac{\sqrt{2}}{288} G_{I J K L}\left(\Gamma_{A}^{I J K L}-8 \delta_{A}^{I} \Gamma^{J K L}\right) \eta \\
& -\frac{1}{1152 \pi}\left(\frac{\kappa}{4 \pi}\right)^{2 / 3} \delta\left(x^{11}\right)\left(\bar{\chi}^{a} \Gamma_{B C D} \chi^{a}\right)\left(\Gamma_{A}^{B C D}-6 \delta_{A}^{B} \Gamma^{C D}\right) \eta+\ldots \\
\delta \psi_{11}= & D_{11} \eta+\frac{\sqrt{2}}{288} G_{I J K L}\left(\Gamma_{11}^{I J K L}-8 \delta_{11}^{I} \Gamma^{J K L}\right) \eta \\
& +\frac{1}{1152 \pi}\left(\frac{\kappa}{4 \pi}\right)^{2 / 3} \delta\left(x^{11}\right)\left(\bar{\chi}^{a} \Gamma_{A B C} \chi^{a}\right) \Gamma^{A B C} \eta+\ldots
\end{aligned}
$$

where gaugino bilinears appear in the right hand side of both expressions. Again we consider gaugino condensation at the hidden $E_{8}$ boundary

$$
\left\langle\bar{\chi}^{a} \Gamma_{i j k} \chi^{a}\right\rangle=g_{8}^{2} \Lambda^{3} \epsilon_{i j k}
$$

The $E_{8}$ gauge coupling constant appears in this equation because the straightforward reduction and truncation leaves a non-canonical normalization for the gaugino kinetic term. An important property of the weakly coupled case ( $d=10$ Lagrangian) was the fact that the gaugino condensate and the three-index tensor field $H$ contributed to the scalar potential in a full square. Hořava made the important observation that a similar structure appears in the M-theory Lagrangian as well [7]:

$$
-\frac{1}{12 \kappa^{2}} \int_{M^{11}} d^{11} x \sqrt{g}\left(G_{A B C 11}-\frac{\sqrt{2}}{32 \pi}\left(\frac{\kappa}{4 \pi}\right)^{2 / 3} \delta\left(x^{11}\right) \bar{\chi}^{a} \Gamma_{A B C} \chi^{a}\right)^{2}
$$

with the obvious relation between $H$ and $G$. Let us now have a closer look at the form of $G$. At the next to leading order we have

$$
\begin{aligned}
G_{11 A B C}= & \left(\partial_{11} C_{A B C}+\text { permutations }\right) \\
& +\frac{1}{4 \pi \sqrt{2}}\left(\frac{\kappa}{4 \pi}\right)^{2 / 3} \sum_{i} \delta\left(x^{11}-x_{i}^{11}\right)\left(\omega_{A B C}^{Y M}-\frac{1}{2} \omega_{A B C}^{L}\right) .
\end{aligned}
$$

Observe, that in the bulk we have $G=d C$ with the Chern-Simons contributions confined to the boundaries. Formula (79) suggests a cancellation between the gaugino condensate and the $G$-field in a way very similar to the weakly coupled case, but the nature of the cancellation of the terms becomes much more transparent now. In the former case we had to argue via the quantization condition for $d B$ that the gaugino condensate is cancelled by one of the Chern-Simons terms. Here this becomes obvious. The condensate is located at the wall as are the Chern-Simons terms, so this 
cancellation has to happen locally at the wall. A quantization condition for $d C$ (the generalization of the quantization condition for $d B$ ) has been discussed in ref. [33].

So this cancellation is very similar to the one in the weakly coupled case. At the minimum of the potential we obtain

$$
G_{A B C 11}=\frac{\sqrt{2}}{32 \pi}\left(\frac{\kappa}{4 \pi}\right)^{2 / 3} \delta\left(x^{11}\right) \bar{\chi}^{a} \Gamma_{A B C} \chi^{a}
$$

at the hidden wall. Substituting this into eqs. (76) and (77) and using Witten's solution [2] (as discussed in section 4.2) we obtain

$$
\delta \psi_{11}=\frac{1}{384 \pi}\left(\frac{\kappa}{4 \pi}\right)^{2 / 3} \delta\left(x^{11}\right)\left(\bar{\chi}^{a} \Gamma_{A B C} \chi^{a}\right) \Gamma^{A B C} \eta+\ldots
$$

This nonzero expectation value of $\delta \psi_{11}$ shows that supersymmetry is spontaneously broken. Because of the cancellation in eq. (79), the cosmological constant vanishes at leading order. Recalling supersymmetry transformation law for the elfbein

$$
\delta e_{I}^{m}=\frac{1}{2} \bar{\eta} \Gamma^{m} \psi_{I}
$$

one finds that the superpartner of the $\mathcal{T}$ field plays the role of the goldstino. Again we have a situation where $F_{\mathcal{S}}=0$ (due to the cancellation in $(\sqrt[79]{70})$ with nonvanishing $F_{\mathcal{T}}$. But here we find the novel and interesting situation that $F_{\mathcal{T}}$ differs from zero only at the hidden wall, although the field itself is a bulk field?. At that wall our discussion is completely 4-dimensional although we are still dealing effectively with a $d=5$ theory. To reach the effective theory in $d=4$ we have to integrate out the dependence of the $x^{11}$ coordinate. As in the previous section this can be performed by the averaging procedure explained there. With the gaugino condensation scale $\Lambda$ sufficiently small compared to the compactification scale $M_{G U T}$, the low-energy effective theory is well described by four dimensional $N=1$ supergravity in which supersymmetry is spontaneously broken. In this case, the modes which remain at low energies will be well approximated by constant modes along the $x^{11}$ direction. This observation justifies our averaging procedure to obtain four dimensional quantities. Averaging $\delta \psi_{11}$ over $x^{11}$, we thus obtain the vev of the auxiliary field $F_{\mathcal{T}}$

$$
F_{\mathcal{T}}=\frac{1}{2} \mathcal{T} \frac{\int d x^{11} \sqrt{g_{1111}} \delta \psi_{11}}{\int d x^{11} \sqrt{g_{1111}}} .
$$

Note that this procedure allows for a nonlocal cancellation of the vev of the auxiliary field in $d=4$. A condensate with equal size and opposite sign at the observable wall could cancel the effect and restore supersymmetry. Using $\int d x^{11} \sqrt{g_{1111}} \delta\left(x^{11}\right)=1$, the auxiliary field is found to be

$$
F_{\mathcal{T}}=\mathcal{T} \frac{1}{32 \pi(4 \pi)^{2 / 3}} \frac{g_{8}^{2} \Lambda^{3}}{R_{11} M_{11}^{3}}
$$

\footnotetext{
${ }^{5}$ In general it would be interesting to consider also situations where the goldstino is not a bulk but a wall field.
} 
Similarly one can easily show that $F_{\mathcal{S}}$ as well as the vacuum energy vanish. This allows us then to unambiguously determine the gravitino mass, which is related to the auxiliary field in the following way:

$$
m_{3 / 2}=\frac{F_{\mathcal{T}}}{\mathcal{T}+\mathcal{T}^{*}}=\frac{1}{64 \pi(4 \pi)^{2 / 3}} \frac{g_{8}^{2} \Lambda^{3}}{R_{11} M_{11}^{3}}=\frac{\pi}{2} \frac{\Lambda^{3}}{M_{\text {Planck }}^{2}} .
$$

As a nontrivial check one may calculate the gravitino mass in a different way. A term in the Lagrangian

$$
-\frac{\sqrt{2}}{192 \kappa^{2}} \int d x^{11} \sqrt{g} \bar{\psi}_{I} \Gamma^{I J K L M N} \psi_{N} G_{J K L M}
$$

becomes the gravitino mass term when compactified to four dimensions. Using the vevs of the $G_{I J K 11}$ given by eq. (81), one can obtain the same result as eq. (86). This is a consistency check of our approach and the fact that the vacuum energy vanishes in the given approximation.

It follows from eq. (86), that the gravitino mass tends to zero when the radius of the eleventh dimension goes to infinity. When the four-dimensional Planck scale is fixed to be the measured value, however, the gravitino mass in the strongly coupled case is expressed in a standard manner, similar to the weakly coupled case as can be seen by inspecting (86) and (75). To obtain the gravitino mass of the order of $1 \mathrm{TeV}$, one has to adjust $\Lambda$ to be of the order of $10^{13} \mathrm{GeV}$ when one constructs a realistic model by appropriately breaking the $E_{8}$ gauge group at the hidden wall.

In the minimization of the potential we have implicitly used the leading order approximation. As was explained in a previous section, the next to leading order correction gives the non-trivial dependence of the background metric on $x^{11}$. Then the Einstein-Hilbert action in eleven dimensions gives additional contribution to the scalar potential in the four-dimensional effective theory, which shifts the vevs of the $G_{I J K L}$. As a consequence, $F_{S}$ will no longer vanish. Though this may be significant when we discuss soft masses, it does not drastically change our estimate of the gravitino mass (86) and our main conclusion drawn here is still valid after the higher order corrections are taken into account.

\section{Soft supersymmetry breaking terms}

In the previous section, we have shown that the gaugino condensation breaks supersymmetry both in the weakly coupled heterotic string and in the heterotic $M$-theory. We chose $\Lambda$ in such a way that the gravitino mass appeared in the TeV-range. In this section we shall discuss the soft supersymmetry breaking terms that appear in the low-energy effective theory as a consequence of this nonzero gravitino mass.

We first give the relevant formulae for gaugino and scalar masses in the observable sector. Given the gauge kinetic function $f_{6}$ in the observable sector, the gaugino mass is calculated to be

$$
m_{1 / 2}=\frac{\partial f_{6}}{\partial \phi^{i}} \frac{F^{i}}{2 \operatorname{Re} f_{6}}
$$


where $\phi^{i}$ symbolically denote hidden sector fields responsible for supersymmetry breakdown. Writing the Kähler potential

$$
K=\hat{K}\left(\phi^{i}, \phi_{i}^{*}\right)+Z\left(\phi^{i}, \phi_{i}^{*}\right) C^{*} C+\left(\text { higher orders in } C, C^{*}\right),
$$

one can also calculate the mass of a matter field $C$ [34, 35]

$$
m_{0}^{2}=m_{3 / 2}^{2}-F^{i} F_{j}^{*} \frac{Z_{i}^{j}-Z_{i} Z^{-1} Z^{j}}{Z}
$$

Here a vanishing cosmological constant is assumed.

Using the classical approximation naively, these formulae lead to a surprising result. All soft masses vanish. At the basis of this fact it had been suggested that the gravitino mass could be arbitrarily high, still leading to softly broken supersymmetry in the TeV range. It has been observed meanwhile that this surprising result is an artifact of the approximation and it is now commonly accepted that generically the soft masses tend to be of the order of the gravitino mass or at least not arbitrarily small compared to it. In general the result for the soft scalar masses is strongly model dependent. We shall see in the following that the situation concerning the gaugino mass is less model dependent but varies when we go from the weakly to the strongly coupled case [3].

\subsection{Weak coupling case}

We start again with the weakly coupled case. At the leading order (tree level), the gauge kinetic function for the observable sector is simply $f_{6}=S$, whereas the gaugino condensation gives $F_{S}=0, F_{T}=m_{3 / 2}\left(T+T^{*}\right)$. Thus, at this level, the gaugino mass vanishes. As was discussed in section 3 , the gauge kinetic function receives corrections at one-loop order. Using eq. (25), the gaugino mass is explicitly written as

$$
m_{1 / 2}=\frac{F_{S}+\epsilon F_{T}}{2 \operatorname{Re}(S+\epsilon T)} .
$$

Note that $F_{T} /\left(T+T^{*}\right) \sim m_{3 / 2}$. Also we expect $F_{S}$ to be of the order of $\epsilon T m_{3 / 2}$ due to the one-loop corrections. Plugging them into the above expression, we obtain

$$
m_{1 / 2} \sim \frac{\epsilon T}{S} m_{3 / 2}
$$

Since in the weakly coupled case the ratio $\epsilon T / S$ is small, the gaugino becomes much lighter than the gravitino.

Let us now consider the scalar masses. At the tree level, the Kähler potential is

$$
K=-\ln \left(S+S^{*}\right)-3 \ln \left(T+T^{*}\right)+\left(T+T^{*}\right)^{n} C^{*} C+\left(\text { higher orders in } C^{*} C\right),
$$

where $n$ denotes the modular weight of a field $C$. For a field with $n=-1$ (untwisted sector in an orbifold construction), which naturally appears in the simple truncation procedure, we recover the previous formula (19). From eq. (90), it follows that

$$
m_{0}^{2}=m_{3 / 2}^{2}+\frac{\left|F_{T}\right|^{2}}{\left(T+T^{*}\right)^{2}}=(1+n) m_{3 / 2}^{2} .
$$


A scalar field with the modular weight -1 has a vanishing supersymmetry-breaking mass at the leading order. It is an artifact of the approximation of reduction and truncation (i.e. torus compactification) that the fields have modular weight -1 . A field whose modular weight is different from -1 has a mass comparable to the gravitino mass. Though, as discussed in section 3, corrections at the one-loop level are model dependent, one expects they are of the order of $\epsilon T / S m_{3 / 2}^{2}$. Summarizing these contributions, one obtains

$$
m_{0}^{2}=(1+n) m_{3 / 2}^{2}+O\left(\frac{\epsilon T}{S} m_{3 / 2}^{2}\right),
$$

where the actual value of the second term depends on the model one considers. A conclusion we can draw from eqs. (92) and (95) is that the gaugino masses tend to be much smaller than the scalar masses:

$$
m_{1 / 2} \ll m_{0} \leq O\left(m_{3 / 2}\right) .
$$

Phenomenologically this relation might be problematic. Requiring that the gaugino masses are at the electro-weak scale, eq. (96) would then imply that the masses of the squarks and sleptons should be well above the $1 \mathrm{TeV}$ region, which raises the fine-tuning problem to reproduce the Fermi scale. Another potential problem is the relic abundance of the lightest superparticles (LSPs) which are likely the lightest neutralinos in the present case. With the parameters characterized by (96), the standard computation of the relic abundances shows that too many LSPs would (if stable) still be around today, resulting in the overclosure of the Universe.

Thus in the weak coupling regime, one can conclude that, though the gaugino condensation realizes the supersymmetry breaking, it tends to lead to a picture where gaugino masses are generically smaller than gravitino and scalar masses. A satisfactory situation might only be achieved, if one fine-tunes the scalar masses in a way that they become comparable to the gaugino masses.

\subsection{Strong coupling case}

Next we want to discuss how the situation changes when one considers the strongly coupled case (heterotic $M$-theory).

As in the weakly coupled heterotic string theory, the gaugino mass vanishes at the leading order of the $\kappa^{2 / 3}$ expansions, because $f_{6}=\mathcal{S}$ and $F_{\mathcal{S}}=0$. Again the next to the leading order is important. The analogue of eq. (91) in the strongly coupled case is

$$
m_{1 / 2}=\frac{F_{\mathcal{S}}+\alpha F_{\mathcal{T}}}{2 \operatorname{Re}(\mathcal{S}+\alpha \mathcal{T})}
$$

Thus we obtain, as before

$$
m_{1 / 2} \sim \frac{\alpha \mathcal{T}}{\mathcal{S}} m_{3 / 2}
$$

A crucial difference in this case, however, is the fact that the ratio $\alpha \mathcal{T} / \mathcal{S}$ is not a small number, but can be as large as unity. This is because the values of $\mathcal{S}$ and $\mathcal{T}$ inferred from our input variables (see section 2.2) suggests that we are rather close to criticality (in which case the ratio becomes unity). Thus we can conclude that, unlike the weakly coupled case, the gaugino mass in the strongly coupled regime is comparable 
to the gravitino mass. This observation confirms the expectation that the gravitino mass should be in the TeV-region and the gaugino condensation scale $\Lambda \sim 10^{13} \mathrm{GeV}$. Because of the simplicity of the mass formula (88) and the fact that the gauge-kinetic function $f$ is stable in higher order perturbation theory, the statement concerning the soft gaugino masses is rather model independent.

The situation is more complicated in the case of the scalar masses which we consider now in the framework of heterotic $M$-theory. At the leading order we arrive at the same conclusions as in the weak coupling case, since the Kähler potential is identical in both cases. In section 4 , we calculated the corrections to the Kähler potential at the next to leading order, which reads

$$
\begin{aligned}
\hat{K} & =-\ln \left(\mathcal{S}+\mathcal{S}^{*}\right)-3 \ln \left(\mathcal{T}+\mathcal{T}^{*}\right) \\
Z & =\frac{6}{\mathcal{T}+\mathcal{T}^{*}}+\frac{2 \alpha}{\mathcal{S}+\mathcal{S}^{*}}
\end{aligned}
$$

where the latter is valid for a field with the modular weight -1 . Now using the formula (90) one may be able to calculate the scalar masses, with the result

$$
\begin{array}{r}
m_{0}^{2}=m_{3 / 2}^{2}-\frac{2-\frac{1}{1+\delta}}{1+\delta} \frac{\left|F_{\mathcal{T}}\right|^{2}}{\left(\mathcal{T}+\mathcal{T}^{*}\right)^{2}}-\frac{\delta\left(2-\delta \frac{1}{1+\delta}\right)}{1+\delta} \frac{\left|F_{\mathcal{S}}\right|^{2}}{\left(\mathcal{S}+\mathcal{S}^{*}\right)^{2}} \\
-\frac{\delta}{(1+\delta)^{2}}\left(F_{\mathcal{S}} F_{\mathcal{T}}^{*}+F_{\mathcal{S}}^{*} F_{\mathcal{T}}\right)
\end{array}
$$

where

$$
\delta \equiv \frac{\alpha}{3} \frac{\mathcal{T}+\mathcal{T}^{*}}{\mathcal{S}+\mathcal{S}^{*}}
$$

We can clearly see from this expression that the structure obtained in the leading order is badly violated. Given the fact that the expansion parameter $\alpha\left(\mathcal{T}+\mathcal{T}^{*}\right) /\left(\mathcal{S}+\mathcal{S}^{*}\right)$ is of order unity it is no longer possible to fine tune the scalar masses (by choosing modular weight -1 for all of them) to a small value and then hope that the corrections respect this fine tuning. In addition the scalar masses depend strongly on the form of the Kähler potential which, in contrast to the gauge kinetic function, receives further corrections in higher order. Thus detailed statements about the scalar masses are very model dependent.

In summary we can, however, conclude with the qualitative statement that in the strong coupling regime,

$$
m_{1 / 2} \sim m_{0} \sim m_{3 / 2}
$$

This contrasts with the relation (96) for the weak coupling regime and represents an important improvement concerning phenomenological applications. In the strongly coupled case, the difference between dilaton- and moduli-dominated supersymmetry breakdown seems less pronounced than it is in the weakly coupled case.

\section{Summary and outlook}

We have presented a consistent framework of supersymmetry breaking and soft breaking terms triggered by the gaugino condensate at the hidden wall. In the strongly coupled 
case, in complete analogy to the weakly coupled case, the gravitino mass $m_{3 / 2}$ is related to the gaugino condensation scale $\Lambda$ as

$$
m_{3 / 2} \approx \frac{\Lambda^{3}}{M_{\text {Planck }}^{2}} .
$$

Furthermore, as explained in section 6 , the soft masses are of the order of the gravitino mass. This implies that these masses should be in the $\mathrm{TeV}$ range in order to solve the naturalness problem of the Higgs boson mass in the supersymmetric framework. This requires that $\Lambda$ should be around $10^{13} \mathrm{GeV}$, three orders of magnitude smaller than the GUT scale (the compactification scale) and thus the 11D Planck scale as well. The gauge coupling constant at the $E_{8}$ wall, where the gaugino condensate is supposed to occur, is larger than the one at the $E_{6}$ wall. If the eleventh dimensional radius $\rho$ approaches the critical radius $\rho_{\text {crit }}$, the $E_{8}$ gauge coupling constant becomes strong at a scale as large as the GUT scale, and the running coupling constant will blow up at that scale already. Then the gaugino condensation scale $\Lambda$, which is approximately identified with the blow-up energy scale, would become too large. For a value of $\Lambda \sim 10^{13} \mathrm{GeV}, \rho$ should (although close) not be too close to the critical value so that the gauge coupling constant does not blow up immediately. This gives a constraint on the constant $\alpha$ (defined in (48)), which depends on the detailed properties of the Calabi-Yau manifold under consideration. In any case it is probably necessary to break the hidden $E_{8}$ to a smaller group to obtain a smaller coefficient of the $\beta$-function. These considerations should be kept in mind when one attempts to construct a realistic model.

The fact that the gravitino mass cannot be arbitrarily large, but should lie in the $\mathrm{TeV}$ range in the heterotic $M$-theory regime suggests that the theory might share a problem already encountered in the weakly coupled case [36, 37, 38]. Late time decay of the gravitinos would upset the success of the standard big-bang nucleosynthesis scenario. This problem is rather universal in most of the supergravity models where breakdown of supersymmetry is mediated through gravity. Indeed this is not really a serious difficulty, but just implies that the universe underwent inflationary expansion followed by reheating at a relatively low temperature $\left(T<10^{9} \mathrm{GeV}\right.$ for $m_{3 / 2}=1 \mathrm{TeV}$ [39]), in which the gravitino number density is diluted by the inflation and the low reheat temperature suppresses gravitino production after that.

A main difference between the weakly and the strongly coupled case manifests itself when we consider phenomenological issues associated with the soft masses. In the weakly coupled string case, the gaugino condensation scenario gives a very small gaugino mass compared to the scalar masses. For a typical size of the compactification radius of the $6 \mathrm{D}$ manifold, the gaugino mass is shown to be more than one order of magnitude smaller than the scalar mass (see for example eqs. (7.20) and (7.24) (with $\sin \theta \rightarrow 0$ limit) of ref. 355 for more detail). This hierarchy among the soft masses obviously raises a naturalness problem. With gaugino masses of the order of $100 \mathrm{GeV}$, the scalar masses would be far above $1 \mathrm{TeV}$, requiring fine tuning to obtain the electroweak symmetry breaking scale. This causes problems for explicit model building. Another phenomenological difficulty caused by the small gaugino mass arises in the context of relic abundances of the lightest superparticles (LSPs). Under the assumption of $R$-parity conservation, the LSP is stable and remains today as a dark matter candidate. Given the superparticle spectrum in the weak coupling regime, the bino, the 
superpartner of the $U(1)_{Y}$ gauge boson, is most likely to be the LSP. To evaluate the relic abundances of the bino, one has to know its annihilation cross section (see ref. 40] and references therein). In our case, the bino pair annihilates into fermion (quarks and leptons) pairs via t-channel scalar (squarks and sleptons) exchange. The cross section is roughly proportional to

$$
\sigma \propto \frac{m_{\tilde{B}}^{2}}{m_{\tilde{f}}^{4}}
$$

where $m_{\tilde{B}}$ is the bino mass and $m_{\tilde{f}}$ represents a scalar mass. As the scalar becomes heavier, the cross section is suppressed, yielding a larger relic abundance. Indeed when the scalar mass is more than an order of magnitude larger than the gaugino mass, a standard calculation shows that the relic abundance exceeds the critical value of the universe. This overclosure is a serious problem in the weakly coupled case.

In the strong coupling regime, the gaugino acquires a mass comparable to the gravitino mass and the scalar masses. Thus the above two problems do not appear. All the soft masses are in the same range. If this is not far from the electroweak scale, one can naturally realize the electroweak symmetry breaking at the correct scale without fine tuning. Moreover in this scenario, the annihilation cross section of the bino becomes larger, and thus we can obtain a relic abundance compatible with the observations. In some regions of parameter space we may even realize a situation where the LSP is the dominant component of the dark matter of the universe.

A characteristic of the mechanism of gaugino condensation is the fact that it is the $T$ field that plays the dominant role in the breakdown of supersymmetry. In this scenario scalar fields with different modular weight will have different masses, which may cause problems with flavor changing neutral currents (FCNC). In the strong coupling case, the situation may be improved through the presence of a large gaugino mass which contributes to the scalar masses at low energies through radiative corrections that can be computed via renormalization group methods. In a situation where scalar masses at the GUT scale are small enough, this universal radiative contribution might wash out nonuniversalities and avoid problems with FCNC. Details of the superparticle phenomenology in the strongly coupled case, including the issues outlined above, will be discussed elsewhere [41].

Eqs. (25) (in the weak coupling case) and (58) (in the strong coupling case) show that the imaginary part of the complex scalar fields, $S$ and $T$, has an axion-like coupling to the gluon fields. In the weakly coupled case, world-sheet instanton effects 42 and possibly other non-perturbative effects give non-negligible contributions to the potential. Then the axion candidates receive masses comparable to the gravitino mass, and they do not solve the strong $C P$ problem. However, in the strongly coupled case, it has been argued that these non-perturbative contributions originated at high energy physics might be suppressed to a negligible level [27, 43, 44. If this is the case, a linear combination of the $\operatorname{Im} \mathcal{S}$ and $\operatorname{Im} \mathcal{T}$ will play a role of the axion, whose potential is dominated by the QCD contribution. Then this axion, referred to as the $M$-theory axion, will be able to solve the strong $C P$ problem. A word of caution should be added here, since a reliable calculation of these world sheet nonperturbative effects has only been performed in the weakly coupled case [19]. The above argumentation in the Mtheory framework uses the implicit assumption that those couplings remain as weak as in the case of the weakly coupled string, an assumption that might not be necessarily 
correct. Apart from that, the axion decay constant in this case becomes as as large as $10^{16} \mathrm{GeV}$, which leads to the potential problem that the energy density of the coherent oscillation of the axion field exceeds the critical energy density of the universe. This problem could be solved if the entropy production occurs after the QCD phase transition when the axion gets massive, or if this world is almost $C P$ conserving and the initial displacement of the axion field is very small. The direct detection of the relic axions with such a large decay constant would be extremely difficult. However the $M$-theory axion may give a significant contribution to the isocurvature density fluctuations during the inflationary epoch, which may be detectable in future satellite observations [45]. It remains to be seen whether this mechanism leads to a satisfactory solution of the strong $\mathrm{CP}$-problem.

In any case we have seen that the M-theoretic version of the heterotic string shows some highly satisfactory phenomenological properties concerning the unification of fundamental coupling constants as well as the nature of the soft supersymmetry breaking parameters.

\section{Acknowledgments}

We would like to thank Jan Conrad for useful discussions. This work was supported by the European Commission programs ERBFMRX-CT96-0045 and CT96-0090 and by a grant from Deutsche Forschungsgemeinschaft SFB-375-95. The work of M.O. was partially supported by the Polish State Committee for Scientific Research grant 2 P03B 040 12. The work of M.Y. was partially supported by the Grant-in-Aid for Scientific Research from the Ministry of Education, Science and Culture of Japan No. 09640333. 


\section{References}

[1] P. Hořava and E. Witten, Nucl. Phys. B460 (1996) 506; Nucl. Phys. B475 (1996) 94.

[2] E. Witten, Nucl. Phys. B471 (1996) 135.

[3] H.P. Nilles, M. Olechowski and M. Yamaguchi, hep-ph/9707143, Phys. Lett. B415 (1997) 24.

[4] J.P. Derendinger, L.E. Ibáñez and H.P. Nilles, Phys. Lett. B155 (1985) 65.

[5] M. Dine, R. Rohm, N. Seiberg and E. Witten, Phys. Lett. B156 (1985) 55.

[6] J.P. Derendinger, L.E. Ibáñez and H.P. Nilles, Nucl. Phys. B267 (1986) 365.

[7] P. Hořava, Phys. Rev. D54 (1996) 7561.

[8] E. Witten, Phys. Lett. B155 (1985) 151.

[9] I. Antoniadis and M. Quirós, Phys. Lett. B392 (1997) 61; hep-th/9705037.

[10] T. Li, J.L. Lopez and D.V. Nanopoulos, hep-ph/9702237; hep-ph/9704247.

[11] E. Dudas and C. Grojean, hep-th/9704177.

[12] E.Dudas, hep-th/9709043.

[13] Z. Lalak and S. Thomas, htp-th/9707223.

[14] A. Lukas, B.A. Ovrut and D. Waldram, hep-th/9710208.

[15] K. Choi, H.B. Kim and C. Muñoz, hep-th/9711158.

[16] A. Lukas, B.A. Ovrut and D. Waldram, hep-th/9711197.

[17] J.O. Conrad, hep-th/9708031.

[18] H. P. Nilles, Lectures at the Trieste Spring School on Supersymmetry, Supergravity and Superstrings 1986, Eds. B. de Wit et al., World Scientific (1986)

[19] J. Lauer, J. Mas and H.P. Nilles, Nucl. Phys. B351 (1991) 353.

[20] M. Shifman and A. Vainshtein, Nucl. Phys. B277 (1986) 456.

[21] H.P. Nilles, Phys. Lett. B180 (1986) 240.

[22] K. Choi and J.E. Kim, Phys. Lett. B165 (1985) 71.

[23] L.E. Ibáñez and H.P. Nilles, Phys. Lett. B169 (1986) 354.

[24] M. Green and J.H. Schwarz, Phys. Lett. B149 (1984) 117.

[25] P. Candelas, G. Horowitz, A. Strominger and E. Witten, Nucl. Phys. B258 (1985) 46. 
[26] H.P. Nilles and S. Stieberger, hep-th/9702110, Nucl. Phys. B499 (1997) 3

[27] T. Banks and M. Dine, Nucl. Phys. B479 (1996) 173.

[28] H.P. Nilles, Phys. Lett. B115 (1982) 193

[29] S. Ferrara, L. Girardello and H.P. Nilles, Phys. Lett. B125 (1983) 457

[30] G.F. Chapline and N.S. Manton, Phys. Lett. B120 (1983) 105.

[31] R. Rohm and E. Witten, Ann. Physics 170 (1986) 454.

[32] H.P. Nilles, Int. Journ. of Modern Physics A5 (1990) 4199.

[33] E. Witten, J. Geom. Phys. 22 (1997) 1.

[34] V.S. Kaplunovsky and J. Louis, Phys. Lett. B306 (1993) 269.

[35] A. Brignole, L.E. Ibáñez and C. Muñoz, Nucl. Phys. B422 (1994) 125.

[36] H. Pagels and J.R. Primack, Phys. Rev. Lett. 48 (1982) 223.

[37] S. Weinberg, Phys. Rev. Lett. 48 (1982) 1303.

[38] J. Ellis, J.E. Kim and D.V. Nanopoulos, Phys. Lett. B145 (1984) 181.

[39] M. Kawasaki and T. Moroi, Prog. Theor. Phys. 93 (1995) 879.

[40] G. Jungman, M. Kamionkowski and K. Griest, Phys. Rep. 267 (1996) 195.

[41] Y. Kawamura, H.P. Nilles, M. Olechowski and M. Yamaguchi, work in progress.

[42] M. Dine, N. Seiberg, X.G. Wen and E. Witten, Nucl. Phys. B289 (1987) 319; Nucl. Phys. B278 (1986) 769.

[43] T. Banks and M. Dine, Nucl. Phys. B505 (1997) 445.

[44] K. Choi, Phys. Rev. D56 (1997) 6588.

[45] M. Kawasaki and T. Yanagida, Prog. Theor. Phys. 97 (1997) 809. 